Article

\title{
Raman and Terahertz Spectroscopic Investigation of Cocrystal Formation Involving Antibiotic Nitrofurantoin Drug and Coformer 4-aminobenzoic Acid
}

\author{
Yong Du ${ }^{1, *}$, Qiang Cai ${ }^{1}$, Jiadan Xue ${ }^{2}$ and Qi Zhang ${ }^{1}$ \\ 1 Centre for THz Research, China Jiliang University, Hangzhou 310018, China; northwestun@163.com (Q.C.); \\ wojiade77tele@163.com (Q.Z.) \\ 2 Department of Chemisty, Zhejiang Sci-Tech University, Hangzhou 310018, China; jenniexue@126.com \\ * Correspondence: yongdu@cjlu.edu.cn; Tel./Fax: +86-571-86875618
}

Academic Editor: Srinivasulu Aitipamula

Received: 1 November 2016; Accepted: 13 December 2016; Published: 16 December 2016

\begin{abstract}
Cocrystallization could improve most physicochemical properties of specific active pharmaceutical ingredients, which has great potential in pharmaceutical development. In this study, the cocrystal of nitrofurantoin and 4-aminobenzoic acid was prepared with solid-state (solvent-free or green-chemistry) grinding approach, and the above cocrystal has been characterized by Raman and terahertz vibrational spectroscopic techniques. Spectral results show that the vibrational modes of the cocrystal within the whole spectral region are different from those of the corresponding parent materials. The dynamic process of such pharmaceutical cocrystal formation has also been monitored directly with Raman spectra. These results offer us unique means for characterizing the cocrystal conformation from the molecule-level, and provides us with rich information about the reaction dynamic of cocrystal formation within pharmaceutical fields.
\end{abstract}

Keywords: nitrofurantoin; active pharmaceutical ingredient; 4-aminobenzoic acid; cocrystallization; intermolecular interaction; Raman spectroscopy; terahertz spectroscopy

\section{Introduction}

Cocrystallization has recently gained great attention as an attractive method to obtain new forms of active pharmaceutical ingredients (APIs) with improved physical and physicochemical properties of drugs [1-6]. Cocrystal design for a specific API is based on evaluating possible synthons with reliable hydrogen bonding motifs (intermolecular interactions) sustaining crystal structures or other non-covalent and nonionic interactions within APIs and cocrystal formers (CCFs). The presence of functional groups acting as hydrogen bond donors and acceptors in the molecules of APIs and CCFs may lead to the formation of cocrystallized substances. Cocrystallization is particularly important in the pharmaceutical field as a result of its impact on drug candidate performance, such as solubility, physical and chemical stability, melting point, and bioavailability of APIs, whereas the pharmacological activity of the APIs is generally not influenced [4,5].

Nitrofurantoin (NF) is a well-known antibacterial drug extensively used as an oral treatment for genitourinary tract infections, and its molecular structure is shown in Figure 1. NF belongs to a biopharmaceutical classification system (BCS) antibiotic drug, and has both low solubility and permeability problems [7-9]. The physicochemical properties, such as the dissolution rate, aqueous solubility, stability, and bioavailability of NF in commercial tablets would decrease due to its polymorphic transformation or photo-degradation during its storage under different extraneous conditions $[7,8]$. These characteristics would significantly impact the therapeutic activity of NF drug. 
Multi-component crystals (also named cocrystals) of NF with various CCFs based on the molecular attributes present have recently been reported [10-14]. The ability of these cocrystals to enhance the photo-stability and clinically-relevant physicochemical properties has been shown in previous works [11-13]. Several cocrystals, such as NF-4-hydroxybenzoic acid, NF-4-aminobenzoic acid, and NF-urea come under the category of pharmaceutical cocrystals [10-17] because of the generally recognized as safe status of the coformers.

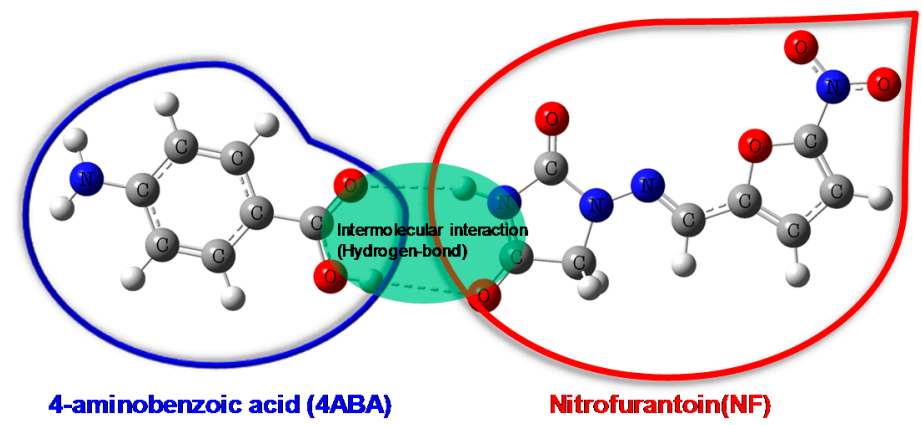

Figure 1. Molecular structures of nitrofurantoin (NF, right) and its coformer 4-aminobenzoic acid (4ABA, left).The green shadow shows the intermolecular interaction due to hydrogen-bond effect between NF and 4ABA molecules.

Differences in crystal structures of cocrystals could be observed and evaluated by lots of research technologies. Traditional techniques that could be employed include powder $\mathrm{X}$-ray diffractormetry (PXRD) [5,18-20], thermal analysis(such as differential scanning calorimetry, DSC), and also solid-state nuclear magnetic resonance spectroscopy (ss-NMR) [20-24]. These established techniques are sometimes time-consuming or lack specific structural information about crystals and/or molecules-especially the effect from the intra-molecular and weak inter-molecular interactions. Vibrational spectroscopic techniques include mid-infrared, Raman, and terahertz (THz) spectroscopy, which have already been successfully used to explain cocrystallization of different solid-state pharmaceuticals [14,24-37]. It is of great importance to use such specific techniques to probe the cocrystal characteristics in order to directly obtain the structural and/or dynamic information at the molecular level to better know the structural information of the specific cocrystal and also its formation mechanism [24,26-28,31,32,35-38].

In this work, Raman and $\mathrm{THz}$ vibrational spectroscopy of a typical cocrystal system formed between NF with cocrystal coformer 4-aminobenzoic acid (4ABA) compounds under solid-state grinding method has been reported. Significant difference among vibrational spectra of the formed cocrystal and involved parent starting molecules is observed. Moreover, the real formation process of NF-4ABA cocrystal could be monitored directly based on the experimental Raman and THz spectral results. The results indicate that vibrational spectroscopy can offer us attractive experimental methods to identify and characterize pharmaceutical cocrystals, and also provide potential tools for further monitoring the real-time reaction dynamics of cocrystals in-line to better know the corresponding reaction mechanism at the molecular level in the pharmaceutical industry.

\section{Results and Discussion}

\subsection{Raman Spectral Characterization of NF, $4 A B A$, and Its Corresponding NF-4ABA Cocrystal}

As a valuable technique for the investigation of solid-state drugs, Raman spectroscopyprovides richintra-molecular structural information and also inter-molecular (e.g., hydrogen bonding effect) interactions between molecules at the molecular level. Raman spectra of NF, 4ABA, their physical mixture, and cocrystal recorded in the wave-number range of $200-1700 \mathrm{~cm}^{-1}$ are shown in Figure 2A,B. 

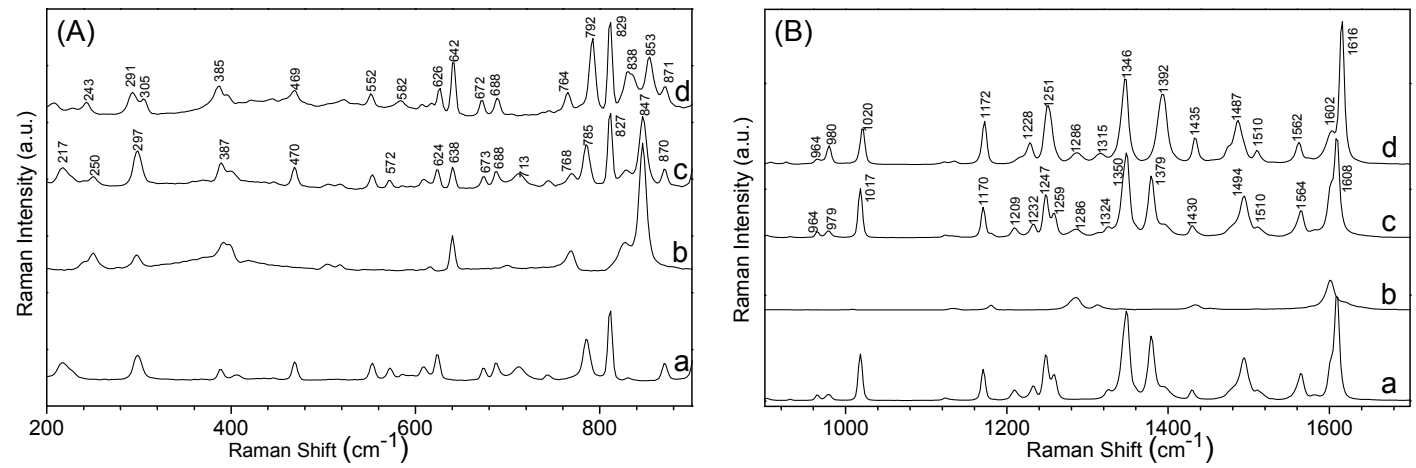

Figure 2. Raman spectra of (a) NF, (b) 4ABA, (c) physical mixture, and (d) cocrystal indifferent spectral regions-(A) 200-900 $\mathrm{cm}^{-1}$; (B) $900-1700 \mathrm{~cm}^{-1}$.

Experimental results show that the Raman spectrum of the physical mixture is almost identical to the linear combination of the individual spectra for the two involved starting components, NF and $4 \mathrm{ABA}$, respectively. It means that there is no intermolecular interaction effect between NF and 4ABA without grinding or other extraneous force. It is well known that the intensities and/or vibrational modes shown in Raman spectra are associated with the change in polarizability of specific chemical bonds within model molecules. Due to the much more rigid and symmetrical structure of the 4ABA molecule, it shows relatively simple Raman bands (which can be seen in Figure $2 b$ ), compared with the Raman spectrum of NF (shown in Figure 2a). Meanwhile, Raman spectra of the corresponding cocrystal formed with grinding method can be easily differentiated from that of physical mixture, which can be observed in Figure 2. Several band shifts and new characteristic features are observed in the whole spectral region, as shown in Figure 2c,d, in which the band locations are given next to characteristic peaks.

Within the spectral region 200-900 $\mathrm{cm}^{-1}$ (most of the vibrational modes in this region are from the deformation of rings and/or in-plane and out-of-plane bending of $\mathrm{C}-\mathrm{H}$ and N-H bonds) shown in Figure 2A, both starting raw materials have band at $297 \mathrm{~cm}^{-1}$. Upon the formation of cocrystal between NF and 4-ABA, this band would split into two peaks at 291 and $305 \mathrm{~cm}^{-1}$, respectively. Some bands (such as 217 and $713 \mathrm{~cm}^{-1}$ ) would disappear, while the band at $838 \mathrm{~cm}^{-1}$, the intensity becomes stronger and the peak width is seen to be much broader after the cocrystallization process. The pure NF Raman spectrum has bands at 785 and $827 \mathrm{~cm}^{-1}$, and the Raman spectrum of pure 4-ABA shows peak at $847 \mathrm{~cm}^{-1}$; however, all three of these characteristic features is up-shifted in the spectrum of the cocrystal at 792,829 , and $853 \mathrm{~cm}^{-1}$, respectively. As for the spectral region from 900 to $1700 \mathrm{~cm}^{-1}$ (most of the vibrational modes in this region are due to stretching and bending vibrations of functional groups; the completed vibrational modes assignment could be found in the Supplementary Materials) shown in Figure 2B, the intensities of 4ABA vibrational modes are pretty weak compared with those of NF. The bands at 1209 and $1269 \mathrm{~cm}^{-1}$ shown in the physical mixture would disappear in cocrystal, and the characteristic bands at 1379 and $1608 \mathrm{~cm}^{-1}$ would be blue-shifted to be at 1392 and $1616 \mathrm{~cm}^{-1}$ upon cocrystal formation. Based on simulation results shown in Table S1 of the Supplementary Materials, the band at $1392 \mathrm{~cm}^{-1}$ is due to stretching vibration of the nitro group of NF and the bending vibration of the carboxylic group within $4 \mathrm{ABA}$; meanwhile, the $1616 \mathrm{~cm}^{-1}$ band comes from the stretching vibration of the carbonyl group within the 4ABA molecule combined with out of plane vibration of the furan ring of the NF molecule.

Raman experimental results indicated that the carbonyl, carboxylic, nitro, and amino groups of $\mathrm{NF}$ and $4 \mathrm{ABA}$ participate in hydrogen bonding formation via inter-molecular interaction during the cocrystallization process. It also confirmed that the crystalline phase of the cocrystal is not simply a combination of the individual starting components, but totally different crystal phase due to the intermolecular interaction effects—especially the hydrogen bonding interaction-between NF and 4ABA molecules. 


\section{2. $T H z$ Spectral Characterization of NF, $4 A B A$, and Its Corresponding NF-4ABA Cocrystal}

The mechanism leading to $\mathrm{THz}$ absorption in molecular systems is dominated by the excitation of intra-molecular as well as inter-molecular vibrations. Therefore, the spectral features in $\mathrm{THz}$ spectra are usually considered to depend on intra-molecular and inter-molecular behavior (such as weak non-covalent bonds like hydrogen bonds or other non-valence bonds). It has especially promising applications in the pharmaceutical field for the analysis of pharmaceutical products throughout the drug development process. Using this technology, it is possible to directly differentiate and quantify different solid-state forms of APIs and their cocrystals, and also exploit structural information for such cocrystals whose molecular and/or crystal structures are stabilized by weak inter-molecular interactions.

$\mathrm{THz}$ absorption spectra of NF, 4ABA, and their physical mixture recorded in the range 0.20-1.70 THz at room temperature are shown in Figure 3, with the $\mathrm{THz}$ signal transmitting through the nitrogen as the reference waveform for the corresponding samples. It can be clearly seen from Figure 3 that both individual components present significantly different absorption features in the recorded range. The absorption bands of $4 \mathrm{ABA}$ centered at $0.61,0.82,0.98,1.34$, and $1.59 \mathrm{THz}$, and the absorption intensity of NF is very weak in the whole frequency range, with a little broad band at around 1.26 and $1.60 \mathrm{THz}$ positions. The contrary intensities of NF and 4ABA shown in above Raman and terahertz spectra indicate that the selection rule of Raman scattering is totally different from that of terahertz absorption effect. Meanwhile, the physical mixture shows the major absorption peaks at the same region, and exhibits some broader than that of $4 \mathrm{ABA}$, which is due to the adding effect from the weak absorption of NF in the same region. The spectrum of the physical mixture is similar to the linear addition of the individual absorption spectra for the two involved starting reactants.

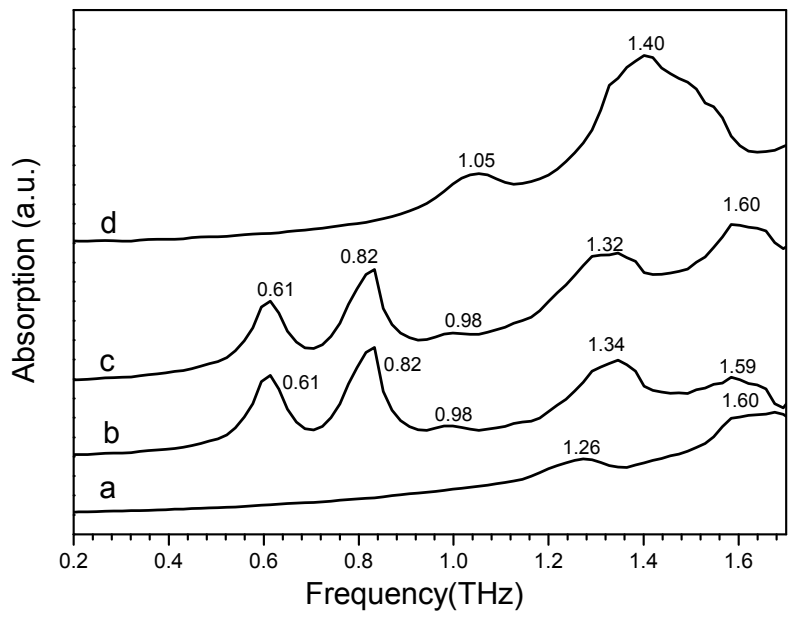

Figure 3. THz spectra of (a) NF; (b) 4ABA; (c) physical mixture; and (d) cocrystal in the $0.2-1.7 \mathrm{THz}$ spectral region.

The spectra of cocrystals obtained from chemical-green grinding methods (shown in Figure 3d) are different compared with those of physical mixture, showing peaks that are not observed in the physical mixture and individual components. The absorption spectra of grinding cocrystal distinguish at frequencies 1.05 and $1.40 \mathrm{THz}$, with broader bands comparing with that of physical mixture. It is well-known that cocrystallization occurs as a result of intermolecular hydrogen bonding, nonionic, or other non-covalent interactions between two or more molecules of the different components [14-16]. So, the experimental results indicate that the vibrational modes observed in these spectra are mostly intermolecular character between $4 \mathrm{ABA}$ and NF molecules during the cocrystal formation process.

The information obtained from $\mathrm{THz}$ spectra is in agreement with that of Raman spectra. It confirms that both Raman and $\mathrm{THz}$ spectroscopy could be promising alternatives to mainstream traditional 
analytical tools such as X-ray diffraction or thermal analysis for further study of solid-state cocrystal reactions in pharmaceutical fields.

\subsection{Dynamic Information during NF-4ABA Cocrystal Formation Process}

To achieve rich dynamic information during cocrystal formation process between NF and $4 \mathrm{ABA}$, the solid-state reactions were performed by neat grinding together equimolar involved parent components with different grinding times. Typical Raman and $\mathrm{THz}$ spectra showing the NF-4ABA cocrystal formation for up to 120 min grinding time can be seen in Figures 4 and 5. Overall, upon grinding, significant changes in both Raman and $\mathrm{THz}$ spectra can be detected with various grinding times, shown in Figures 4 and 5.

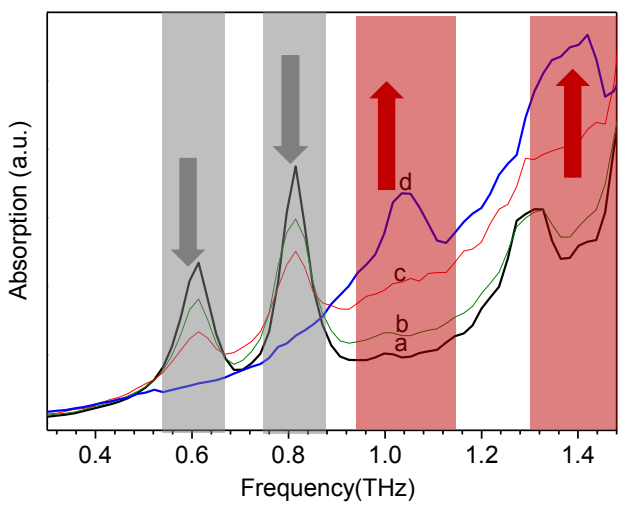

Figure 4. THz spectral comparison of the formed cocrystal between NF and 4ABA using grinding method under typical reaction times. (a) $0 \mathrm{~min}$; (b) $15 \mathrm{~min}$; (c) $30 \mathrm{~min}$; (d) $80 \mathrm{~min}$. The shadowed area shows the typical $\mathrm{THz}$ band change during the grinding process.
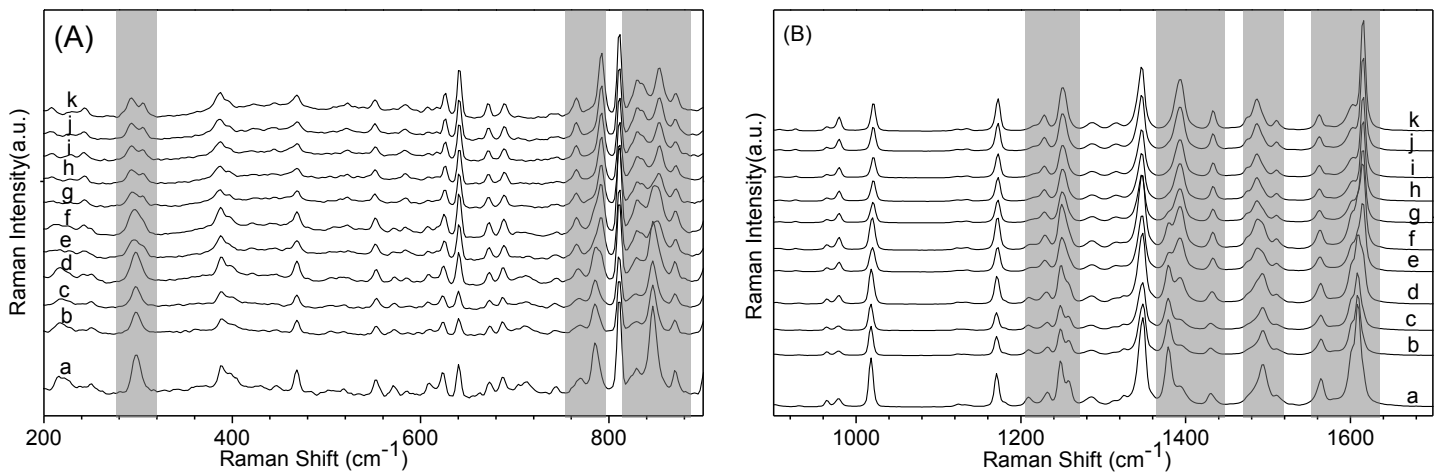

Figure 5. Raman spectral comparison of the formed cocrystal between NF and 4ABA using grinding method under typical reaction times in different spectral regions-(A) $200-900 \mathrm{~cm}^{-1}$; (B) $900-1700 \mathrm{~cm}^{-1}$. (a) $0 \mathrm{~min}$, (b) $5 \mathrm{~min}$, (c) $15 \mathrm{~min}$, (d) $25 \mathrm{~min}$, (e) $30 \mathrm{~min}$, (f) $40 \mathrm{~min}$, (g) $50 \mathrm{~min}$, (h) $60 \mathrm{~min}$, (i) $70 \mathrm{~min}$, (j) $80 \mathrm{~min}$, (k) $120 \mathrm{~min}$. The shadowed area shows the typical Raman band change during the grinding process.

In $\mathrm{THz}$ spectral comparison of the formed NF-4ABA cocrystal shown in Figure 4, the intensity of the characteristic absorption peak of the physical mixture (at $0.61,0.82$, and $1.34 \mathrm{THz}$, shown with grey arrows) decreases, while that of the cocrystal peaks (at 1.05 and $1.40 \mathrm{THz}$ ) increases gradually with typical grinding time during its formation process. There are two clear isobestic points appearing at around 0.67 and $0.87 \mathrm{THz}$ in Figure 4, which indicates the change of absorption peaks due to physical mixture and cocrystal in the reaction process. It means that the starting parent mixture was consumed while a continuation of the cocrystal formation takes place. After around 120 min's grinding time, the $\mathrm{THz}$ absorption spectrum only shows characteristic peaks of cocrystal, and characteristic 
features from the physical mixture (e.g., $0.61,0.82 \mathrm{THz}$ ) disappear completely, indicating a thorough cocrystallization between NF and 4ABA.

According to Raman spectral result, the evolution of cocrystal characteristic peaks could also be clearly observed with increasing grinding time shown in Figure 5 with grey arrows, meanwhile, the intensity of characteristic peaks of NF and $4 \mathrm{ABA}$ is accompanied by a decrease or even disappearance. The more the grinding time performed, the higher the characteristic vibrational peaks of NF-4ABA cocrystal are obtained. This demonstrates that the longer-time grinding process might strongly induce and promote the cocrystallization transformation between NF and 4ABA. The Raman spectrum of cocrystal formation at $80 \mathrm{~min}$ is almost same as that of even longer $120 \mathrm{~min}$, and it indicates that the solid-state reaction is already completed at around $80 \mathrm{~min}$ of grinding time, and this result is also consistent with that of above THz spectra shown in Figure 4.

Due to the strong baseline influence and also pretty broad band shown in the $\mathrm{THz}$ spectral results, it is not easy to reveal the accurate dynamic informationwith quantitative analysis for cocrystal transformation between NF and 4ABA. While, Raman spectra are good choice to obtain such information because of its better signal-noise ratio and also pretty weak baseline influence within the whole spectral region seen in Figure 5. Cocrystal formed from NF and 4ABA has characteristic Raman spectral features that could be used for quantification to obtain dynamic information so that the reaction mechanism could be achieved during the cocrystallization process. Further analysis of the tentative changes in the Raman spectra shown in Figure 5 may reveal the dynamics of the NF-4ABA cocrystal formation with different grinding times.

Two Raman bands at 1379 and $1392 \mathrm{~cm}^{-1}$ (shown in Figure 5B)—which are characterized for the physical mixture and cocrystal, respectively - are chosen as an indicator. The dynamic information about time-dependent cocrystallization process of NF and 4ABA could be deduced by fitting the data between the normalized relative peak areas and grinding times with single exponential functions. The decay rate from the physical mixture's characteristic peak $\left(\tau_{1379} \approx 25.5 \mathrm{~min}\right)$ and the growth rate of cocrystal $\left(\tau_{1392} \approx 26.0 \mathrm{~min}\right.$ ) are consistent with each other, which indicates the successive promotion of the conversion of NF-4ABA cocrystal from the starting raw materials. Such first-order transformation during NF-4ABA cocrystallization process is pretty agreement well with previous works from Friscic et al. [37] and Emmerling et al. [24], and there is no intermediate appearance within NF-4ABA formation process. From the tentative changes shown in Figure 6, such solid-state pharmaceutical cocrystal transformation process and the corresponding dynamic information can be directly quantified and investigated.

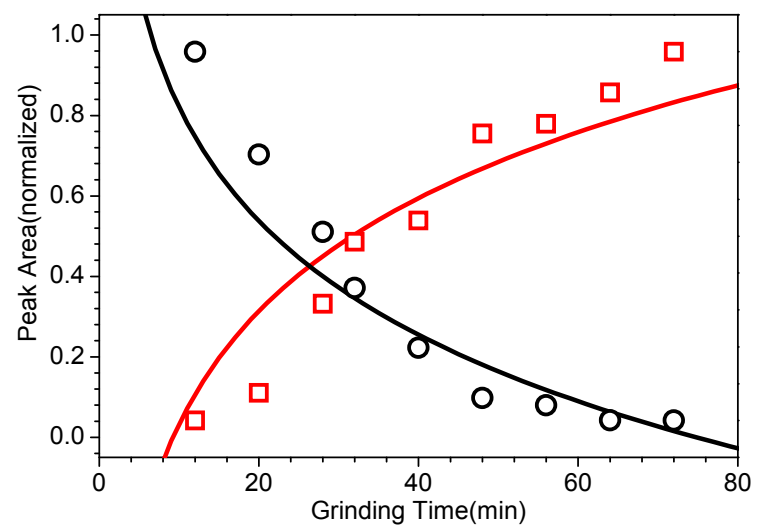

Figure 6. Observed normalized change of the relative peak area for the features at $1379 \mathrm{~cm}^{-1}$ (characterized feature for the physical mixture, shown with black circle and black fitting-curving line) and $1392 \mathrm{~cm}^{-1}$ (characterized feature for the cocrystal, shown with red square and red fitting-curving line) as the grinding time progresses. 


\section{Materials and Methods}

NF and 4ABA samples were purchased from Sigma-Aldrich (Sigma-Aldrich, Shanghai, China) (structures shown in Figure 1) and used without further purification. Starting materials with a total load of $2 \mathrm{~g}$ were weighed, and its physical mixture was obtained by gently mixing two compounds above at a 1:1 molar ratio in a glass vial by using a vortex mixer. Grinding cocrystal was performed by neat co-grinding NF and $4 \mathrm{ABA}$ physical mixture in $25 \mathrm{~mL}$ stainless steel milling jars using a planetary ball mill (QM-3SP, gear type, Nanjing University Instrument Plant, Nanjing, China) with a frequency of $25 \mathrm{~Hz}$ and twenty $5 \mathrm{~mm}$ steel balls at room temperature. A measured amount of product produced in the solid-state reaction at different grinding times was taken out for further spectra analysis.

All the samples were weighted into $\sim 150 \mathrm{mg}$ aliquots and poured into a steel die and subjected to $\sim 4 \mathrm{MPa}$ pressure for several minutes. The sample discs $\sim 13 \mathrm{~mm}$ in diameter, $\sim 1.5 \mathrm{~mm}$ thick were obtained and sealed in plastic bags before spectroscopic analysis.

Raman spectra of all samples were obtained using Fourier Transform Raman spectrometer (Thermo Nicolet 960, Waltham, MA, USA) with diode pumped $1064 \mathrm{~nm}$ solid-state laser as the near-IR source. Spectra were acquired over 500 scans at $2 \mathrm{~cm}^{-1}$ resolution.

Terahertz time-domain spectroscopy (THz-TDS) measurement was performed with a commercial Zomega-2 time-domain THz spectrometer (Zomega Terahertz Corp., Troy, NY, USA). The THz beam is produced by a Ti:Sapphire oscillator ultrafast laser with a $75 \mathrm{MHz}$ repetition rate, $780 \mathrm{~nm}$ center wavelength, and $\sim 100 \mathrm{fs}$ pulse duration. The $\mathrm{THz}$ region of the spectrometer was purged with nitrogen gas to reduce the absorption effect from ambient water vapor. A total of three $\mathrm{THz}$ spectra (representing three complete sets of sample and reference measurements) were averaged for each final spectrum. The time-domain of the $\mathrm{THz}$ electric field was recording for the reference (without sample) and each sample. $\mathrm{THz}$ absorption spectra were obtained with the fast Fourier transform (FFT) operation.

\section{Conclusions}

Using Raman and THz-TDS spectroscopy, Raman scattering, and THz absorption spectra of NF, 4ABA, the corresponding physical mixture, and also cocrystals formed from chemical-green grinding methods have been recorded. Both spectra obtained in the measurement region provide finger-print information of starting raw components and cocrystals, which may be useful for the discrimination of these different solid-state pharmaceutical molecular systems. The dynamic process of NF-4ABA cocrystal formation can also be monitored directly with Raman and THz spectra. From the analysis of tentative changes in Raman spectra, such solid-state pharmaceutical cocrystallization transformation reaction and the corresponding kinetics information can be directly quantified and investigated. The reported results offer us the unique means and benchmark to identify and characterize the cocrystal conformation at the molecular level, and further also real-time monitor the reaction dynamic process of cocrystals in pharmaceutical fields.

Supplementary Materials: The following are available online at www.mdpi.com/2073-4352/6/12/164/s1, Figure S1: Molecular structure of the cocrystal between nitrofurantoin (NF) and 4-aminobenzoic acid(4ABA); Figure S2: Raman vibrational modes of the cocrystal between NF and 4ABA. (a) theoretical calculation based on form C shown in Figure S1; (b) experimental spectrum; Table S1: Vibrational modes assignment for Raman characteristic peaks of the cocrystal between NF and 4ABA.

Acknowledgments: This work was supported partly by the National Natural ScienceFoundation of China (Grant No. 21205110, 21202032), and Natural Science Foundation of Zhejiang Province (Grant Nos. LY15B50004, LY16B030008).

Author Contributions: Yong Du, Qiang Cai and Qi Zhang conceived and designed the experiments; Yong Du, Qiang Cai, Jiadan Xue and Qi Zhang performed the experiments; Yong Du and Jiadan Xue analyzed the data and wrote the paper.

Conflicts of Interest: The authors declare no conflict of interest. 


\section{References}

1. Vishweshwar, P.; McMahon, J.A.; Bis, J.A.; Zaworotko, M.J. Pharmaceutical co-crystals. J. Pharm. Sci. 2006, 95, 499-517. [CrossRef] [PubMed]

2. Friscic, T.; Jones, W. Benefits of cocrystallisation in pharmaceutical materials science: An update. J. Pharm. Pharmacol. 2010, 62, 1547-1559. [CrossRef] [PubMed]

3. Chieng, N.; Rades, T.; Aaltonen, J. An overview of recent studies on the analysis of pharmaceutical polymorphs. J. Pharm. Biomed. Anal. 2011, 55, 618-644. [CrossRef] [PubMed]

4. Qiao, N.; Li, M.; Schlindwein, W.; Malek, A.; Davies, A.; Trappitt, G. Pharmaceutical cocrystals: An overview. Int. J. Pharm. 2011, 419, 1-11. [CrossRef] [PubMed]

5. Liao, X.; Gautam, M.; Grill, A.; Zhu, H. Effect of position isomerism on the formation of pharmaceutical cocrystals. J. Pharm. Sci. 2010, 99, 246-254. [CrossRef] [PubMed]

6. Hasa, D.; Rauber, G.S.; Voinovich, D.; Jones, W. Cocrystal formation throughmechanochemistry: From Neat and liquid-assisted grinding to polymer-assisted grinding. Angew. Chem. Int. Ed. 2015, 54, 7371-7375. [CrossRef] [PubMed]

7. Tian, F.; Qu, H.; Louhi-Kultanen, M.; Rantanen, J. Mechanistic insight into the evaporative crystallization of two polymorphs of nitrofurantoin monohydrate. J. Cryst. Growth 2009, 31, 2580-2589. [CrossRef]

8. Aaltoene, J.; Strachan, C.J.; Pollanen, K.; Yliruusi, J.; Rantanen, J. Hyphenated spectroscopy as polymorph screening tool. J. Pharm. Biomed. Anal. 2007, 44, 477-483. [CrossRef] [PubMed]

9. Sardo, M.; Amado, A.M.; Ribeiro-Claro, P. Hydrogen bonding in nitrofurantoin polymorphs: A computation-assisted spectroscopic study. J. Raman Spectrosc. 2009, 40, 1956-1965. [CrossRef]

10. Vangala, V.R.; Chow, P.S.; Tan Reginald, B.H. Characterization, physicochemical and photo-stability of a co-crystal involving and antibiotic drug, nitrofurantoin, and 4-hydroxybenzoic acid. CrystEngComm 2011, 13, 759-762. [CrossRef]

11. Vangala, V.R.; Chow, P.S.; Tan Reginald, B.H. Co-crystal and co-crystal hydrates of the antibiotic nitrofurantoin: Structural studies and physicochemical properties. Cryst. Growth Des. 2012, 12, 5925-5938. [CrossRef]

12. Alhalaweh, A.; George, S.; Basavoju, S.; Childs, S.L.; Rizvi, S.A.; Velaga, S.P. Pharmaceutical cocrystals of nitrofurantoin: Screening, characterization and crystal structure analysis. CrystEngComm 2012, 14, 5078-5088. [CrossRef]

13. Cherukuvada, S.; Babu, N.J.; Nangia, A. Nitrofurantoin-aminobenzoic acid cocrystal: Hydration stability and dissolution rate studies. J. Pharm. Sci. 2011, 100, 3233-3244. [CrossRef] [PubMed]

14. Zhang, Q.; Fang, H.X.; Zhang, H.L.; Qin, D.; Hong, Z.; Du, Y. Co-crystal between nitrofurantion and urea investigated by terahertz spectroscopy and density functional theory. Acta Chim. Sin. 2015, 73, 1069-1073.

15. Tutughamiarso, M.; Bolte, M.; Wagner, G.; Egert, E. Five pseudopolymorphs and a cocrystal of nitrofurantoin. Acta Crystallogr. C 2011, 67, o18-o25. [CrossRef] [PubMed]

16. Vangala, V.R.; Chow, P.S.; Tan, B.H. The solvates and salt of antibiotic agent, nitrofurantoin: Structural, thermochemical and desolvation studies. CrystEngComm 2013, 15, 878-889. [CrossRef]

17. Wang, H.; Xiao, H.; Liu, N.; Zhang, B.; Shi, Q. Three new compounds derived from nitrofurantoin: X-ray structures and Hirshfeld surface analyses. Open J. Inorg. Chem. 2015, 5, 63-73. [CrossRef]

18. Lin, Y.; Yang, H.; Yang, C.; Wang, J. Preparation, characterization, and evaluation of dipfluzine-benzoic acid co-crystals with improved physicochemical properties. Pharm. Res. 2014, 31, 566-578. [CrossRef] [PubMed]

19. Rehder, S.; Klukkert, M.; Lobmann, K.A.M.; Stracha, C.J.; Sakmann, A.; Gordon, K.; Rades, T.; Leopold, C.S. Investigation of the formation process of two piracetam cocrystals during grinding. Pharmaceutics 2011, 3, 706-722. [CrossRef] [PubMed]

20. Sowa, M.; Slepokura, K.; Matczakjon, E. A 1:1 pharmaceutical cocrystal of myricetin in combination with uncommon piracetam conformer: X-ray single crystal analysis and mechanochemical synthesis. J. Mol. Struct. 2014, 1058, 114-121. [CrossRef]

21. Ghosh, S.; Bag, P.P.; Reddy, C.M. Cocrystals of sulfamethazine with some carboxylic acids and amides. Cryst. Growth Des. 2011, 11, 3489-3503. [CrossRef]

22. Limwikrant, W.; Higashi, K.; Yamamoto, K.; Moribe, K. Characterization of ofloxacin-oxalic acid complex by PXRD NMR and THz spectroscopy. Int. J. Pharm. 2009, 382, 50-55. [CrossRef] [PubMed] 
23. Zhang, G.C.; Lin, H.L.; Lin, S.Y. Thermal analysis and FTIR spectral curve-fitting investigation of formation mechanism and stability of indomethacin-saccharin cocrystals via solid-state grinding process. J. Pharm. Biomed. Anal. 2012, 66, 162-169. [CrossRef] [PubMed]

24. Kulla, H.; Greiser, S.; Benemann, S.; Rademann, K.; Emmerling, F. In situ investigation of a self-accelerated cocrystalformation by grinding pyrazinamide with oxalic acid. Molecules 2016, 21, 917. [CrossRef] [PubMed]

25. Brittain, H.G. Vibrational spectroscopic studies of cocyrstal and salts 1: Benzamide-benzoic acid system. Cryst. Growth Des. 2009, 9, 2492-2499. [CrossRef]

26. Brittain, H.G. Vibrational spectroscopic studies of cocyrstal and salts 2: Benzylamine-benzoic acid system. Cryst. Growth Des. 2009, 9, 3497-3503. [CrossRef]

27. Soares, F.L.F.; Carneiro, R.L. Green synthesis of ibuprofen-nicotinamide cocrystals and in-line evaluation by Raman spectroscopy. Cryst. Growth Des. 2013, 13, 1510-1517. [CrossRef]

28. Yan, J.; Li, S.; Zhao, H.; Song, B.; Zhang, G.; Zhang, J.; Zhu, Y.; Han, J. Molecular recognition and interaction between uracil and urea in solid-state studied by terahertz time-domain spectroscopy. J. Phys. Chem. A 2014, 118, 10927-10933.

29. Nguyen, K.L.; Friscic, T.; Day, G.M.; Gladden, L.F.; Jones, W. Terahertz time-domain spectroscopy and the quantitative monitoring of mechanochemical cocrystal formation. Nat. Mater. 2007, 6, 206-210. [CrossRef] [PubMed]

30. Shen, Y.C. Terahertz pulsed spectroscopy and imaging for pharmaceutical applications: A review. Int. J. Pharm. 2011, 417, 48-60. [CrossRef] [PubMed]

31. Du, Y.; Fang, H.X.; Zhang, Q.; Zhang, H.L.; Hong, Z. Spectroscopic investigation on cocrystal formation between adenine and fumaric acid based on infrared and Raman techniques. Spectrochim. Acta A Mol. Biomol. Spectrosc. 2016, 153, 580-585. [CrossRef] [PubMed]

32. Du, Y.; Zhang, H.L.; Xue, J.D.; Fang, H.X.; Zhang, Q.; Xia, Y.; Li, Y.; Hong, Z. Raman and terahertz spectroscopical investigation of cocrystal formation process of piracetam and 3-hydroxybenzoic acid. Spectrochim. Acta A Mol. Biomol. Spectrosc. 2015, 139, 488-494. [CrossRef] [PubMed]

33. Du, Y.; Xia, Y.; Zhang, H.L.; Hong, Z. Using terahertz time-domain spectroscopical technique to monitor cocrystal formation between piracetam and 2,5-dihydroxybenzoic acid. Spectrochim. Acta A Mol. Biomol. Spectrosc. 2013, 111, 192-195. [CrossRef] [PubMed]

34. Fang, H.X.; Zhang, Q.; Zhang, H.L.; Qing, D.; Hong, Z.; Du, Y. Terahertz spectroscopic and density functional theoretical analysis of cocrystal between olmesartan and benzoic acid. Chem. J. Chin. Univ. 2015, 36, 2504-2510.

35. Fang, H.X.; Zhang, Q.; Zhang, H.L.; Du, Y.; Hong, Z. Terahertz spectroscopic analysis of adenine and fumaric acid cocrystals. Acta Phys. Chim. Sin. 2015, 31, 221-226.

36. Du, Y.; Zhang, H.L.; Xue, J.D.; Tang, W.; Fang, H.X.; Zhang, Q.; Li, Y.; Hong, Z. Vibrational spectroscopic study of polymorphism and polymorphic transformation of the anti-viral drug lamivudine. Spectrochim. Acta A Mol. Biomol. Spectrosc. 2015, 137, 1158-1163. [CrossRef] [PubMed]

37. Gracin, D.; Strukil, V.; Friscic, T.; Halasz, I.; Uzarevic, K. Laboratory real-time and in situ monitoring of mechanochemical milling reactions by Raman spectroscopy. Angew. Chem. Int. Ed. 2014, 53, 6193-6197. [CrossRef] [PubMed]

38. Du, Y.; Xue, J.D. Investigation of polymorphism and cocrystallization of active pharmaceuticaling redients using vibrational spectroscopic techniques. Curr. Pharm. Des. 2016, 22, 4917-4928. [CrossRef] [PubMed]

(C) 2016 by the authors; licensee MDPI, Basel, Switzerland. This article is an open access article distributed under the terms and conditions of the Creative Commons Attribution (CC-BY) license (http://creativecommons.org/licenses/by/4.0/). 